Research Article

\title{
Genetically Modified Porcine Mesenchymal Stem Cells by Lentiviral Tbx18 Create a Biological Pacemaker
}

\author{
Yannan Hu, ${ }^{1}$ Ning Li $\mathbb{D}^{1},{ }^{1}$ Liang Liu, ${ }^{1,2}$ Hao Zhang, ${ }^{1}$ Xiang Xue, ${ }^{3}$ Xin Shao, ${ }^{1}$ Yu Zhang, \\ and Xilong Lang iD 1 \\ ${ }^{1}$ Department of Cardiothoracic Surgery, Changhai Hospital, Second Military Medical University, Shanghai, China \\ ${ }^{2}$ Department of Thoracic Surgery, Changzhou TCM Hospital, Changzhou, China \\ ${ }^{3}$ Department of Cardiothoracic Surgery, The Affiliated Second Hospital, Suzhou University, Suzhou, China
}

Correspondence should be addressed to Xilong Lang; langxl2017@163.com

Received 3 April 2019; Revised 13 July 2019; Accepted 24 August 2019; Published 7 November 2019

Academic Editor: Ji-Dong Fu

Copyright (c) 2019 Yannan Hu et al. This is an open access article distributed under the Creative Commons Attribution License, which permits unrestricted use, distribution, and reproduction in any medium, provided the original work is properly cited.

\begin{abstract}
Background. Tbx18 is a vital transcription factor involved in embryonic sinoatrial node (SAN) formation process but is gradually vanished after birth. Myocardial injection of lentiviral Tbx18 converts cardiomyocytes into pacemaker-like cells morphologically and functionally. In this in vitro and in vivo study, genetical modification of porcine bone mesenchymal stem cells (BMSCs) by recapturing the Tbx18 expression creates a biological pacemaker which was examined. Methods. The isolated porcine BMSCs were transfected with lentiviral Tbx18, and the induced pacemaker-like cells were analyzed using real-time polymerase chain reaction and western blotting to investigate the efficiency of transformation. Then, the induced pacemaker-like cells were implanted into the right ventricle of the SAN dysfunction porcine model after the differentiation process. Biological pacemaker activity and ectopic pacing region were tested by an electrocardiograph (ECG) monitor. Results. The isolated porcine BMSCs expressed specific surface markers of stem cells; meanwhile, the expression of myocardial markers was upregulated significantly after lentiviral Tbx18 transfection. The porcine SAN dysfunction model was constructed by electrocoagulation using a surgical electrotome. The results showed that the mean heart beat (HR) of BMSCs-Tbx18 was significantly higher than that of BMSCsGFP. An ectopic pacing region was affirmed into the right ventricle by ECG after implantation of BMSCs-Tbx18. Conclusion. It was verified that Lenti-Tbx18 is capable of transducing porcine BMSCs into pacemaker-like cells. Genetically modified porcine BMSCs by lentiviral Tbx18 could create a biological pacemaker. However, further researches in large-scale animals are required to rule out unexpected complications prior to application in clinical practice.
\end{abstract}

\section{Introduction}

At present, electronic pacemakers have become the standard treatment for bradyarrhythmia, including sick sinus syndrome (SSS) and atrioventricular block. It is reported that SSS is the predominant indication for pacemaker implantation in the United States, and the incidence of SSS increases with age. Hence, the number of these patients will increase sharply over the next 50 years and inevitably burden the medical budget [1]. The application of pacemakers has significantly improved the life quality of these patients. However, it should be noted that implanted electronic pacemakers can only alleviate the symptoms related to bradyarrhythmia while accompanied with some complications, including bleeding, infection, and battery exhaustion. In addition, pacemakers merely guarantee the basic physiological needs for these patients on account of that the pacing parameters are usually preset as certain values, which can hardly meet the requirements under various conditions.

Gene and cell therapies spark the development of biological pacemakers, which may address such limitations [2]. It is well known that the sinoatrial node (SAN) is the origin of cardiac electrical activity and sets the rhythm for the heart. SAN is different from other cardiac tissue in that its electric potential can be automatically depolarized 
at hyperpolarization. Therefore, a biological pacemaker can be achieved by restoring pacing ability of SAN or creating an ectopic pacing region.

One prevalent approach to acquire a biological pacemaker is genetic reprogramming of ventricular myocytes by a viral vector, including the embryonic transcription factors related to SAN formation and ion channel-associated proteins, to create an ectopic pacing region [3]. For example, the T-box (Tbx) gene family, including Tbx3 and Tbx18, plays an important role in the embryonic formation of SAN, and the short stature homeobox transcription factor 2 (Shox2) is also involved in this developmental process [4-6]. Moreover, ion channel-associated proteins, mainly hyperpolarization-activated cyclic nucleotide-gated channel 4 (HCN4), are related to the diastolic depolarization process of SAN [7]. In vitro and in vivo studies of gene manipulation for rodent neonatal rat cardiomyocytes to restore the ectopic pacing region by overexpressing embryonic transcription factors and ion channel-associated proteins have been proved to be successful [8-12]. Kapoor et al. have proved that Tbx18 was the most effective transcription factor with the ability to transform neonatal ventricular myocytes into SAN-like cells by transducing a panel of transcription factors individually, including Shox2, Tbx3, Tbx5, Tbx18, and Tbx20 [11]. It is worth mentioning that these reconstructed cells are similar to native SAN cells in both morphology and physiological automaticity [11]. Tbx18 has also been used to induce pacemaker-like cells from somatic myocytes in situ in a large-scale animal model of complete heart block $[13,14]$. However, adenovirus-based genetic manipulation cannot maintain its function more than 2-4 weeks, which hinders its clinical feasibility.

Another avenue to biological pacing is gene- and cellbased hybrid treatment, which can be achieved by loading stem cells with the pacemaker gene for prolonged gene expression duration. Stem cells are optimal candidate carriers for their self-renewal and multipotent differentiation ability. Phenotypic transformation of BMSCs into pacemaker-like cells mediated by Tbx18 has been verified in the mRNA and protein level [15-17]. Besides, both Shox 2 and Tbx 3 have also been confirmed to enhance the differentiation efficacy of stem cells into pacemaker-like cells [18-20]. However, those studies in large-scale animal models are limited.

To the best of our knowledge, previous investigations mainly focused on inducing pacemaker-like cells via genetic reprogramming of somatic cardiomyocytes or evaluating the possibility for acquiring pacemaker-like cells by oriented differentiation from stem cells. In this article, we created a biological pacemaker by recapturing the Tbx18 expression of BMSCs in vitro and in a large-scale animal model. To this end, this study may provide a new genetic and cellular therapy for the treatment of bradyarrhythmia.

\section{Materials and Methods}

2.1. Porcine BMSC Isolation and Cell Culture. Porcine bone marrow was harvested aseptically into a $15 \mathrm{ml}$ conical tube and suspended in $5 \mathrm{ml}$ phosphate-buffered solution (PBS). The buffy coat was isolated by centrifugation $(1500 \mathrm{rpm}$,
$20 \mathrm{~min}$ ) using $5 \mathrm{ml}$ Ficoll (GE Healthcare, USA). The purified cells were collected and washed twice in sterile PBS [21]. The supernatant was removed after centrifugation, and then, the cells were resuspended and planked into 6-well plates with proper cell density. BMSCs were cultured in Dulbecco's modified Eagle's medium (Gibco, USA) supplemented with $100 \mathrm{U} / \mathrm{ml}$ penicillin (Gibco, USA), $100 \mu \mathrm{g} / \mathrm{ml}$ streptomycin (Gibco, USA), and 10\% FBS (Gibco, USA) at $37^{\circ} \mathrm{C}$ under a humidified $5 \%$ carbon dioxide incubator. The culture medium was refreshed every 2 days.

2.2. Construction of the Tbx18 Lentiviral Vector. PLentiCMV-EGFP (Lenti-GFP) (OBiO Tech Co. Ltd., Shanghai, China) was digested using EcoR I and BamH I. The ORF sequence of the human Tbx18 gene (OBiO Tech Co. Ltd., Shanghai, China) was amplified using polymerase chain reaction (PCR). A restriction enzyme reaction procedure was performed before gel extraction. The digested gene fragment and vector were integrated to form pLenti-CMVTbx18-EGFP (Lenti-Tbx18), which was then transferred into competent $\mathrm{DH} 5 \alpha$ cells (OBiO Tech Co. Ltd., Shanghai, China). Positive clones were identified by sequencing. Bacteria were incubated in a shaker at $37^{\circ} \mathrm{C}$ overnight in LB culture medium. Recombinant plasmids were extracted using a Plasmid Midi Preparation Kit (Beijing ComWin Biotech Co. Ltd., Beijing, China). HEK 293T cells were transfected with LentiTbx18 and backbone vectors using Lipofectamine 2000 (Invitrogen, USA). The Lenti-GFP was packaged as a control. The supernatant was harvested after viral amplification. The acquired Lenti-Tbx18 and Lenti-GFP were stored at $-80^{\circ} \mathrm{C}$.

2.3. BMSCs Transfected with Tbx18. When BMSCs reached $70-80 \%$ confluency, lentiviral Tbx18 was used to transfect the cells at a multiplicity of infection (MOI) of 100. These transfected BMSCs were used as the experimental group; meanwhile, the BMSCs treated with Lenti-GFP were treated as the control. The culture medium was replaced with fresh complete medium after $6 \mathrm{~h}$ transfection procedure. These cells were observed using a fluorescent microscope to determine the transfection rate after $24 \mathrm{~h}$. At last, these cells were cultured for an additional 7 days for morphological observation.

2.4. Flow Cytometry Analysis. The isolated BMSCs were collected by trypsinization, centrifuged at $1200 \mathrm{rpm}$ for $5 \mathrm{~min}$, washed by PBS twice, and then transferred into $1.5 \mathrm{ml} \mathrm{EP}$ tubes. The supernatant was discarded after centrifugation. BMSCs were blocked by BSA (Thermo, USA) solution for $30 \mathrm{~min}$. Then, BMSCs were incubated with primary antibodies CD29 (ab6124, 1:400), CD90 (ab23894, 1:100), CD34 (ab81289, 1:100), and CD45 (ab10558, 1:400) overnight at $4^{\circ} \mathrm{C}$, respectively. BMSCs without incubation with the primary antibody were set as a negative control. Then, the corresponding FITC-labeled secondary antibodies were added for $1 \mathrm{~h}$ at room temperature in the dark. Cells were centrifuged at $1500 \mathrm{rpm}$ for $5 \mathrm{~min}$, and the supernatant was removed. Cells were washed with PBS twice and resuspended in PBS prior to analysis using a flow cytometer. 
2.5. mRNA Real-Time Quantitative PCR (RT-qPCR). Total RNA was extracted by a TRIzol Reagent kit (Takara, Japan) following the manufacturer's protocol. The concentration was determined by spectrophotometry at $260 \mathrm{~nm}$. For mRNA quantification, $1 \mu \mathrm{g}$ of total RNA was reversetranscribed to cDNA using the PrimeScript RT reagent kit (Takara, Japan). The SYBR Green RT-PCR Kit (Takara, Japan) and LightCycler 480 System (Roche, Switzerland) were used for quantitative RT-PCR analysis. The mRNA expression was normalized to the reference gene GAPDH for each cDNA sample. Amplification conditions were set as follows: $95^{\circ} \mathrm{C}$ of preincubation for $3 \mathrm{~min}, 40$ cycles of denaturation at $95^{\circ} \mathrm{C}$ for $30 \mathrm{~s}$, annealing at $62^{\circ} \mathrm{C}$ for $30 \mathrm{~s}$, and final extension at $72^{\circ} \mathrm{C}$ for $30 \mathrm{~s}$. All results were normalized to GAPDH expression levels and compared to the expression level at baseline by the $2^{-\Delta \Delta C t}$ method. The primers used in this experiment are presented in Table 1.

2.6. Western Blotting. After each treatment, cells were washed with PBS and lysed in SDS buffer containing a protease inhibitor cocktail $(1: 100)$ on ice for 30 minutes. Total protein concentrations were evaluated using a protein assay kit (Beyotime Biotech, Shanghai, China). A cell lysate was separated using 10\% SDS-PAGE and then transferred to polyvinylidene difluoride membranes. Subsequently, the membranes were blocked for $1 \mathrm{~h}$ at room temperature by incubation in TBST containing 5\% $(w / v)$ nonfat milk. Primary antibodies against HCN4 (ab32675, $1: 5000)$, cTnI (ab47003, 1:500), $\alpha$-SA (ab156302, $1: 1000)$, Tbx18 (ab115262, 1:800), and GAPDH (Proteintech, $1: 5000$ ) were incubated overnight at $4^{\circ} \mathrm{C}$. Finally, the membranes were incubated with corresponding secondary antibodies for 1 hour at room temperature and detected by an ECL Chemiluminescent Substrate Reagent Kit (Thermo, USA) and exposed to a film.

2.7. Immunofluorescence Staining. The myocardial tissue at the injection site was fixed with $4 \%$ paraformaldehyde. Then, a routine three-step protocol was conducted: sectioning, dewaxing, and hydrating tissues. Following permeabilization with $0.1 \%$ Triton $\mathrm{X}-100$, the tissue was incubated with a primary anti-Tbx18 antibody (ab115262, 1:200) overnight at $4^{\circ} \mathrm{C}$. A secondary antibody with green fluorescence was then used to detect Tbx18. We used 4',6-diamidino-2-phenylindole (DAPI) to visualize the nuclei. The cells were observed under a fluorescence microscope.

2.8. Large-Scale Animal Experiment. Adult Yorkshire pigs (male, $40 \mathrm{~kg}$ ) were managed with conformation to the "Shanghai Administration Rule of Laboratory Animal," and the Institutional Animal Care and Use Committee approved the protocol of the Animal Care Center at the Second Military Medical University. Firstly, the pig was sedated by injection of midazolam $(0.25 \mathrm{mg} / \mathrm{kg})$, supplied with ketamine $(8 \mathrm{mg} / \mathrm{kg})$ intramuscularly; then, the pig was constrained on the animal operating table and endotracheally intubated. Electrocardiograph (ECG) leads were inserted into the 4 limbs subcutaneously and connected to the data acquisition (DAQ) system for continuous signal collection. Mechanical
TABle 1: Primer sequences used in the study.

\begin{tabular}{lc}
\hline Gene & \multicolumn{1}{c}{ Primer sequences } \\
\hline Tbx18 & Forward: ACCCTCAACCGATACAGCAC \\
& Reverse: GACATTCCCGAAATCTGCAT \\
GAPDH & Forward: GAGTCAACGGATTTGGTCGT \\
& Reverse: TTGATTTTGGAGGGATCTCG \\
\hline
\end{tabular}

ventilation was performed for proper perioperative management and prevention of pneumothorax. Subsequently, anesthesia was maintained by propofol $(4 \mathrm{mg} / \mathrm{kg} / \mathrm{h}$, intravenously). Right $4^{\text {th }}$ intercostal mini-incision was employed to provide straight access to the right heart and minimize operative wound. The pericardium was opened using surgical scissors and suspended on the chest wall by sutures for facilitating the surgical procedure. Two pacing leads were implanted onto the surface of the right ventricle and adjacent subcutaneous tissue beforehand, which was a vital safeguard procedure in case of emergency of malignant arrhythmia during operation. At last, SAN was destroyed by highfrequency electric knifes with energy of $60 \mathrm{~J}$. The injection site was located at the right ventricle without fat covering. BMSCs-Tbx18 or BMSCs-GFP were implanted in the injection site $\left(300 \mu \mathrm{l}\right.$ per injection, 3 injections per pig, $4 \times 10^{6}$ cells per injection).

2.9. Statistical Analysis. Data are expressed as the mean \pm standard deviation, and statistical analysis was performed with GraphPad software. Normally distributed variables were analyzed using the Student $t$-test or paired $t$-test, and nonnormally distributed variables were analyzed using the Wilcoxon signed rank test. $P$ value $<0.05$ was considered statistically significant for all tests.

\section{Results}

3.1. Cell Characteristics of BMSCs and Demonstration of Gene Transfer. Figure 1(a) shows the result of surface markers expressed in the isolated BMSCs, which are identified by flow cytometric analysis. The positive expression of stromal cell markers (CD29 and CD90) coupled with the absence of hematopoietic markers (CD45 and CD34) indicates that these cells were purified BMSCs. The result of negative control is shown in Figure S1. BMSCs exhibit spindlelike morphology after adherence as shown in Figure 1(b). When the cell confluence reached $80 \%$, BMSCs were transfected with Lenti-Tbx18 and corresponding LentiGFP, respectively. Green fluorescence could be detected after transfection for $24 \mathrm{~h}$ (Figure 1(c)). In addition, optimal infection efficiency can be achieved when the multiple of infection (MOI) was set at 100. Figures $1(\mathrm{~d})$ and $1(\mathrm{e})$ vividly show the morphological change of BMSCs after transfection for 7 days. BMSCs with an original spindle shape gradually transformed into a strip shape, which is a typical feature of SAN cells [22]. In addition, the beating was observed in approximately 7\% BMSCs-Tbx18 at day 10 (approximately $90 \mathrm{bpm}$ ), and the beating could last for about 3 weeks in vitro. 


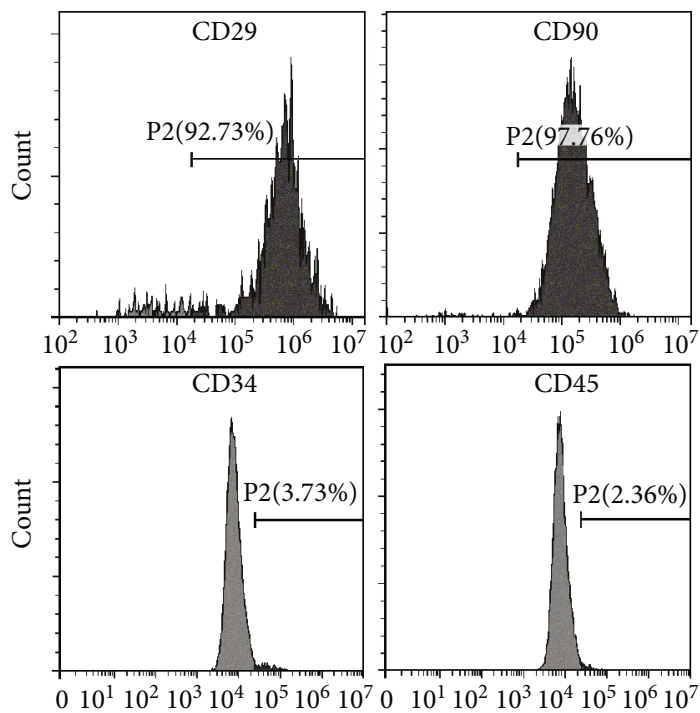

(a)

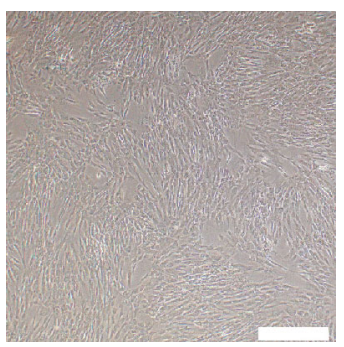

(b)

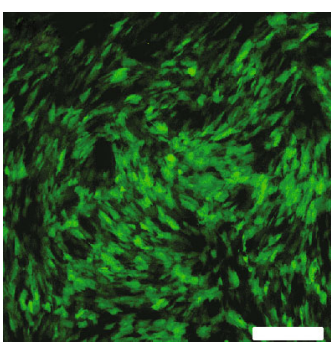

(c)

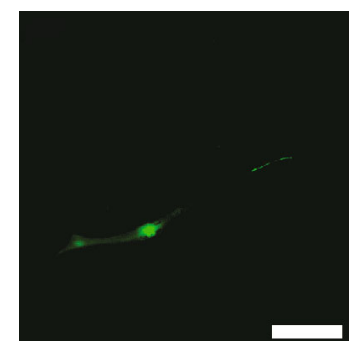

(d)

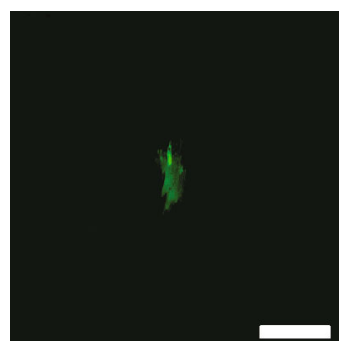

(e)

Figure 1: Cell characteristics of BMSCs and demonstration of gene transfer. (a) Surface marker of BMSCs under flow cytometry. BMSCs: bone mesenchymal stem cells; CD: cluster of differentiation. (b) Cellular morphological feature of BMSCs exhibiting a spindle shape. Scale bar $=400 \mu \mathrm{m}$. (c) Green fluorescence of BMSCs after transfection with Lenti-Tbx18 for $48 \mathrm{~h}$. Scale bar $=400 \mu \mathrm{m}$. (d, e) Representative morphological characterizations of BMSCs with a strip shape and original spindle shape after transduction with Lenti-Tbx18 and Lenti-GFP for 7 days, respectively. Scale bar $=200 \mu \mathrm{m}$.

3.2. Tbx18 Overexpression in BMSCs Promotes BMSCs Differentiation into Pacemaker-Like Cells. The abovementioned morphological change suggests that BMSCs may differentiate into pacemaker-like cells after transduction by Lenti-Tbx18. While molecular evidence is also needed for further confirmation of such differentiation process. The expression level of Tbx18 is significantly upregulated, which is demonstrated by RT-qPCR and western blotting (Figures 2(a) and 2(b)). At the same time, the protein expression levels of $\alpha$-SA, cTnI, and HCN4 were assessed by western blotting as shown in Figures 2(c) and 2(d). The results indicated that $\alpha$-SA, cTnI, and HCN4 expression levels in the BMSC-Tbx18 group were significantly upregulated. Taking the morphological alteration and molecular change together, Tbx18 overexpression in BMSCs promotes BMSC differentiation into pacemaker-like cells.

3.3. Model Building of SAN Dysfunction in Pig. The SAN, which is located at the junction of the superior vena cava and the right atrium, sets the rhythm for the heart. Figure 3(a) shows the photograph of the model building procedure for SAN dysfunction by a high-frequency electrotome. 9 pigs were used to construct SAN dysfunction model, 1 died of massive hemorrhage on account of superior vena cava rupture. Real-time ECG monitoring was necessary to assess the effect of model building. Normal ECG, including regular $\mathrm{P}$ waves, followed by QRS complex, and T waves, is shown in Figure $3(\mathrm{c})$. The mean heart rate $(\mathrm{HR})$ is significantly reduced after the model building process (Figure 3(b)). The absence of $\mathrm{P}$ wave accompanied with arrhythmia (Figure 3(d)) verified that a SAN dysfunction model in large-scale animals can be successfully conducted by electrocoagulation using a surgical electrotome.

3.4. Tbx18 Biological Pacemaker Enhances Heart Rate with Favorable Autonomic Respondence. Figure 4(a) shows the implantation procedure of transduced BMSCs into the right ventricle. Meanwhile, a temporary pacemaker was employed to prevent the occurrence of malignant bradycardia arrhythmia and set as a positive control for cardiac pacing. The mean HR of the BMSC-Tbx18 group is significantly higher than that of the BMSC-GFP group in day 2 after injection and persisting for continuous 5 weeks as shown in Figures 4(b) and 4(c). In addition, the mean $\mathrm{HR}$ reaches its maximum value at the $2^{\text {nd }}$ week and then trends down. The HR of the BMSC-Tbx18 group maintains at a higher level than that of 


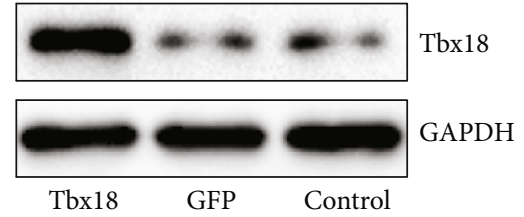

(a)

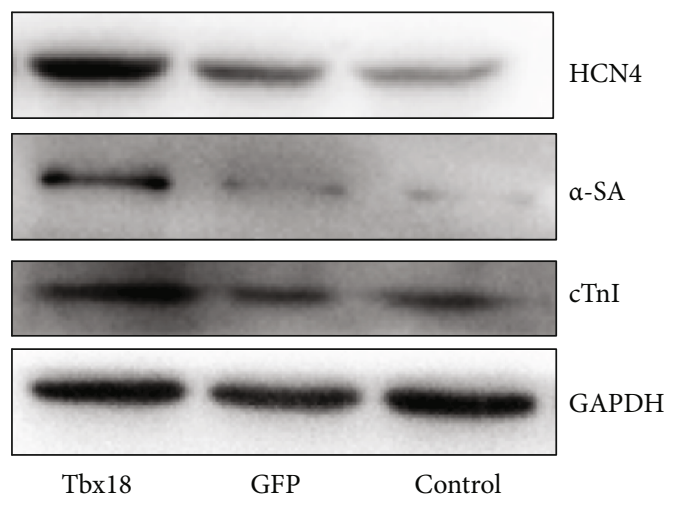

(c)

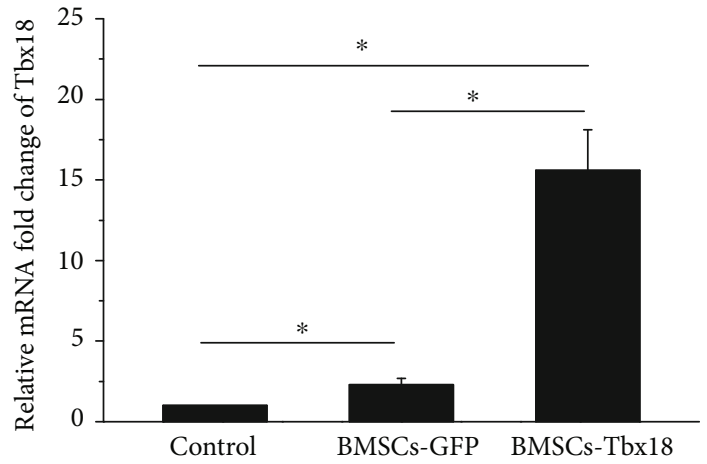

(b)

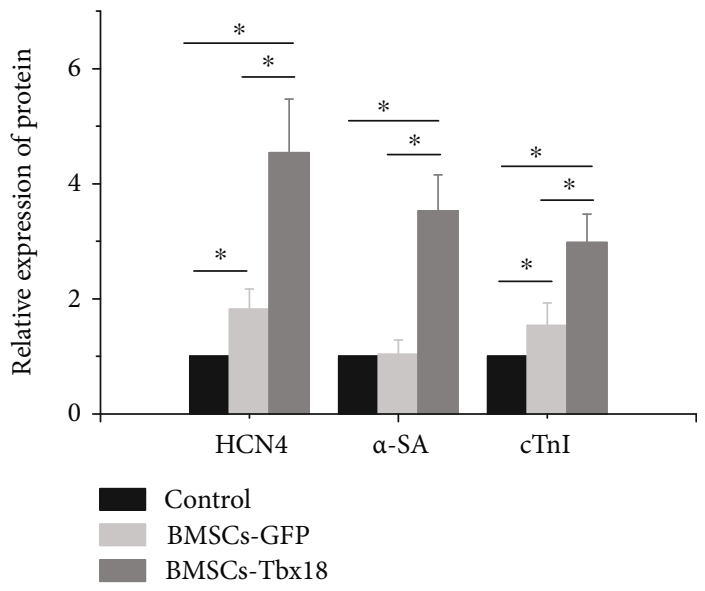

(d)

FIGURE 2: Western blotting and PCR analysis of target protein and mRNA expression level after transfection. (a) Western blotting of Tbx18 expression level after transfection for $72 \mathrm{~h}$. BMSCs without transfection are set as the control. (b) PCR analysis of Tbx 18 expression level after transfection for 48 h. * represents $P<0.05$. (c) Western blotting detects increased HCN4, $\alpha$-SA, and cTnI protein expression. (d) Quantitative analysis of the protein expression levels of HCN4, $\alpha$-SA, and cTnI. * represents $P<0.05$. cTnI: cardiac troponin I; GFP: green fluorescent protein; HCN4: hyperpolarization-activated cyclic nucleotide-gated channel $4 ; \alpha$-SA: $\alpha$-striated actin.

the BMSC-GFP group at all time points of our study. Since the poor engraftment and unwanted migration will inevitably occur during the implantation process, the immunofluorescence test was used to verify the existence of BMSCs-Tbx18 in the injection site at the end of the 5-week experiment. The positive expression of Tbx18 indicates that BMSCsTbx18 can be observed after 5 weeks as shown in Figure S2. Figure 4(d) shows that isoproterenol infusion $(3 \mu \mathrm{g} / \mathrm{kg} / \mathrm{h})$ for $10 \mathrm{~min}$ increased HR by $72 \%$ and $65 \%$ in the BMSCTbx18 group and BMSC-GFP group, respectively. The active reaction to the $\beta$-adrenergic agonist indicates that the Tbx18 biological pacemaker may provide an avenue to address the shortcoming of insufficient response to neurotransmitter signals for the electronic pacemaker.

\subsection{BMSC-Tbx18 Biological Pacemaker Activity Originates} from the Right Ventricle. To investigate the origin site of cardiac electrical activity, we use the DAQ system to capture and analyze ECG instantaneously. As we know, the electronic pacemaker is fabricated to stimulate the contraction of ventricles by simulating the function of SAN through releasing electrical stimuli periodically. Cardiac pacing can be achieved by inserting a pacing lead into the right ventricular endocardium intravenously for permanent application or suturing a pacing lead on the surface of the right ventricle for temporary use. Figures 5(a) and 5(d) show typical ECG of electronic pacing, in which a pacer spike followed by a prolonged QRS complex can be observed. Regular prolonged QRS complexes without corresponding pacer spike in the BMSC-Tbx18 group indicated that pacing signals were derived from the right ventricle (Figures 5(b) and 5(e)). In contrast, no ectopic electrical signal was observed in the BMSC-GFP group (Figures 5(c) and 5(f)). Together, these results support that Tbx18-induced pacemaker activity was released from the right ventricle.

\section{Discussion}

Previous studies have proposed the notion that Tbx18 could significantly enhance the converting efficiency of mature cardiac myocytes into pacemaker-like cells. Notably, the porcine cardiomyocytes were demonstrated to be transformed into 


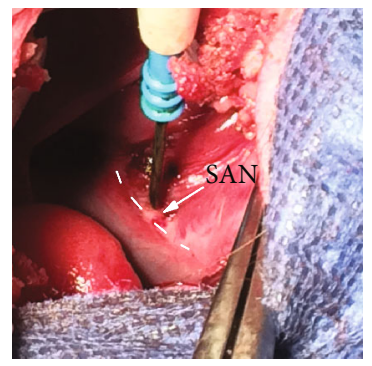

(a)

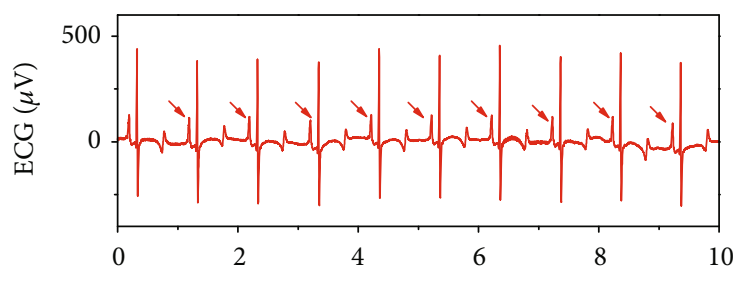

(c)

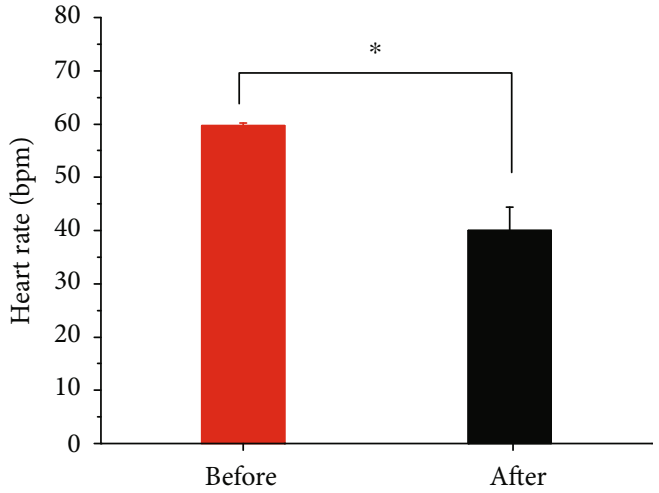

(b)

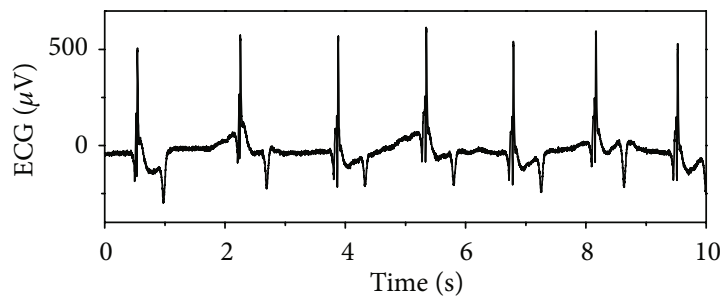

(d)

FIgURE 3: Model building of SAN dysfunction in pig. (a) Photograph of the construction procedure for SAN dysfunction by high-frequency electrotome. White arrow represents the location of SAN. (b) Mean heart rate of pigs before and after the model construction procedure. Data are means \pm SEM. bpm: beats per minute. * represents $P<0.05$. (c) Representative ECG of sinus rhythm before the model construction procedure. Red arrow represents $\mathrm{P}$ wave. (d) Representative ECG of arrhythmia after the model construction procedure.

pacemaker-like cells morphologically and functionally, in which the ectopic pacing site can maintain their function for nearly 2 weeks [14]. However, this issue remains controversial since no aberrant expression of SAN-specific genes could be observed in the chamber myocardium of fetal mice after Tbx18 reexpression [23]. In addition, pathological structural abnormalities, including right ventricular hypoplasia, atrial dilatation, and ventricular septal defects, were observed in this procedure. The induced pacemaker-like cells have been reported to be with high cAMP level, which is related to their function while they may affect numerous genes regulated by cAMP-dependent phosphorylation, leading to undesirable effects like neoplasm [24, 25].

In this article, we successfully create a biological pacemaker with longer operating time by modifying porcine BMSCs with Lenti-Tbx18 and verify its potential in the porcine SAN dysfunction model, which is consistent with the previous reports that Tbx18 can be used as a candidate for biological pacing.

The expression level of $\mathrm{HCN}$ is significantly upregulated after transduction of Tbx18, which is identical to previous results and may be related to aberrant pacemaker-like cell formation $[15,16,26,27]$. It is reported that autodepolarization of SAN cells at hyperpolarization is due to the funny current $\left(I_{\mathrm{f}}\right)$, which is driven by $\mathrm{HCN}$ protein $[7,28,29]$. HCN mutations may be associated with multiple families of bradycardia and SAN dysfunction, which indicates that HCN may play a vital role in maintaining stable function of SAN [30, 31]. Previous animal experiments have demonstrated that overexpression of HCN4 in the porcine heart can significantly reduce the dependence on pacemakers; furthermore, an ectopic pacing region can be induced by implanting BMSCs loaded with HCN4 [9, 32-34].

In addition, several procedures to construct a SSS animal model have been introduced in previous research, primarily involving physical impairment of the SAN, including condensation, radiofrequency ablation, and ligation of the right coronary artery. While in this paper, we successfully construct a model of SAN dysfunction by surgical technique under direct vision with a higher achievement ratio. However, a pig died of massive hemorrhage since the superior vena cava was ruptured by electrocoagulation, which suggests that a safer model building method should be further explored. Sodium hydroxide or formaldehyde wet compression has been successfully applied in small animal models with lower death rate, which may provide some hints for model building in large-scale animals [35-37]. A combination of physical and chemical methods may provide a new model building way with a higher achievement ratio and lower rate of complications.

In conclusion, we successfully create a biological pacemaker with longer operating time by modifying porcine BMSCs with Lenti-Tbx18 and verify its potential in the porcine SAN dysfunction model. This study may provide a novel alternative treatment for bradyarrhythmia. However, Tbx 18 overexpression may lead to some unexpected complications, which requires further research in large-scale animals prior to application in clinical practice. 


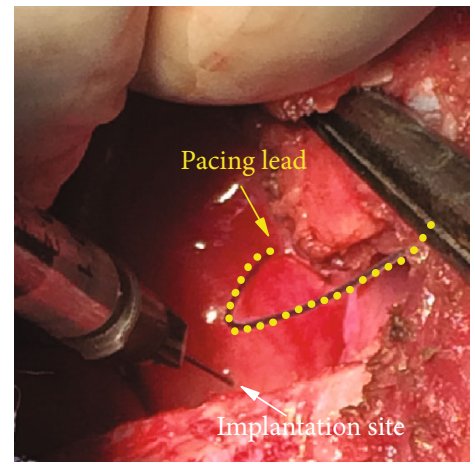

(a)

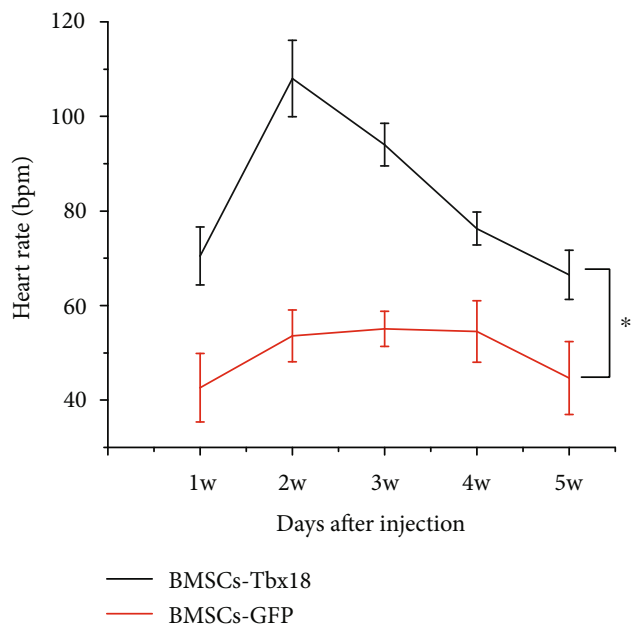

(c)

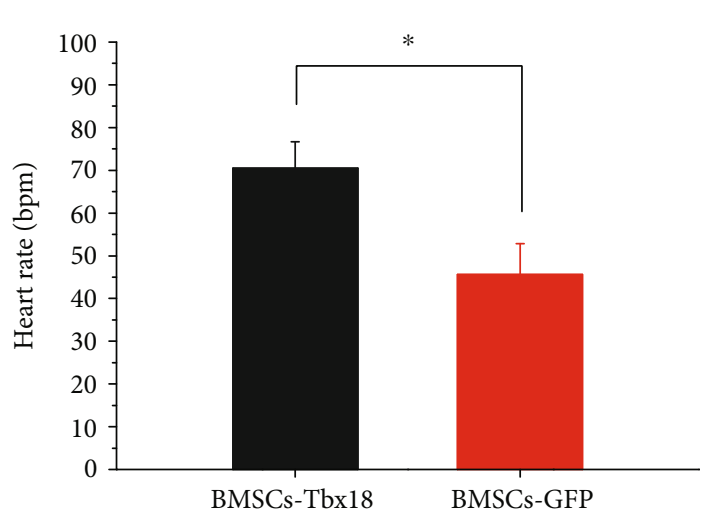

(b)

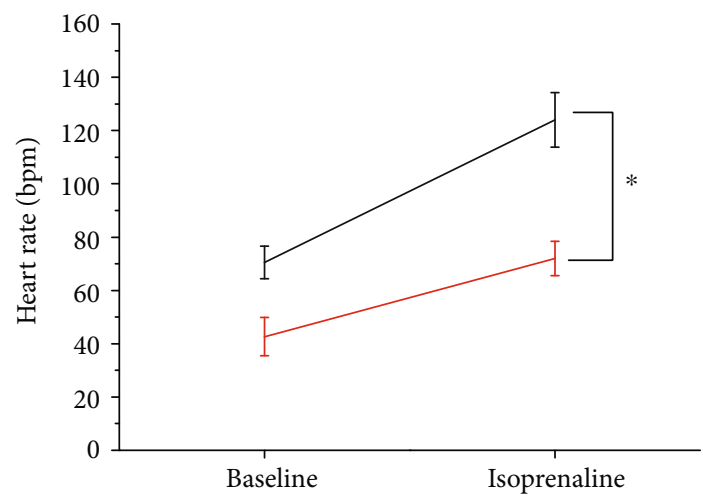

(d)

Figure 4: BMSCs-Tbx18 create a biological pacemaker. (a) Photograph of the implantation procedure for transduced cells. Yellow arrow represents the pacing lead of the temporary epicardial pacemaker, and white arrow represents the implantation site of the right ventricle. (b) Mean heart rate of the BMSC-Tbx18 group and BMSC-GFP group after injection for 2 days. Data are means \pm SEM. ${ }^{*} P<0.05$ compared to the BMSC-GFP group. (c) Mean heart rate of both groups after injection for continuous 5 weeks. Data are means \pm SEM. ${ }^{*} P<0.05$ compared to the BMSC-GFP group. (d) Increasement in heart rate after continuous isoproterenol infusion for 10 min between both two animal groups. ${ }^{*} P<0.05$ compared to the BMSC-GFP group.

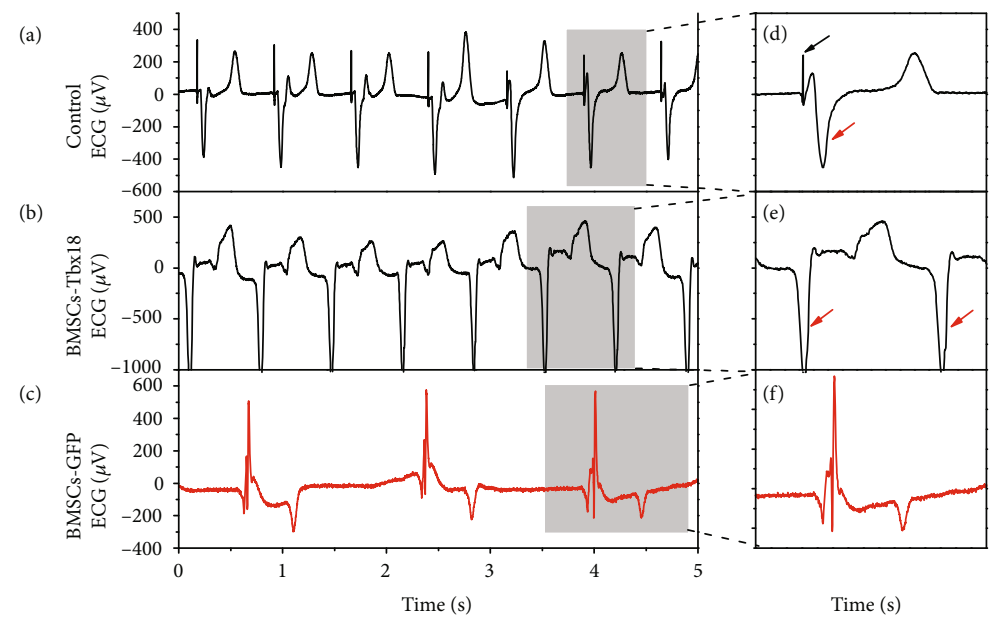

FIGURE 5: BMSC-Tbx18 biological pacemaker activity originates from the right ventricle. (a-c) Representative ECG of the temporary epicardial pacemaker, BMSC-Tbx18 group, and BMSC-GFP group; the pacing rate of the epicardial pacemaker was preset at $80 \mathrm{bpm}$ as a control. (d-f) Enlarged figure of corresponding ECG for the temporary epicardial pacemaker, BMSC-Tbx18 group, and BMSC-GFP group; red arrows represent the prolonged QRS complex (duration > $0.12 \mathrm{~s}$ ), and black arrow represents the ventricular pacer spike. 


\section{Data Availability}

The original data used to support the findings of this study are available from the corresponding author upon request.

\section{Conflicts of Interest}

The authors declare that there are no conflicts of interest.

\section{Authors' Contributions}

YN. H., N. L., and L. L. performed all of the experimental works and data analysis, and H. Z., X. X., X. S., and Y. Z. drafted the manuscript. XL. L. designed and conducted the study and revised the manuscript. Yannan $\mathrm{Hu}$, $\mathrm{Ning} \mathrm{Li}$, and Liang Liu contributed equally to this work.

\section{Acknowledgments}

This study was funded by the National Key Research and Development Program of China (No. 2016YFC1100900) and the Second Military Medical University Innovation Fund for Doctor (No. 20172038).

\section{Supplementary Materials}

Figure S1: the flow cytometry histograms of the negative control group. Figure S2: laser confocal microscopy of immunofluorescence staining for Tbx18 in the rejection site. (A) Confocal microscopy image showing positive expression of TBX18 (green fluorescence). 4',6-diamidino2-phenylindole (DAPI) indicates nuclei. Scale bar, $50 \mu \mathrm{m}$. (Supplementary Materials)

\section{References}

[1] P. N. Jensen, N. N. Gronroos, L. Y. Chen et al., "Incidence of and risk factors for sick sinus syndrome in the general population," Journal of the American College of Cardiology, vol. 64, no. 6, pp. 531-538, 2014.

[2] M. Farraha, S. Kumar, J. Chong, H. Cho, and E. Kizana, "Gene therapy approaches to biological pacemakers," Journal of Cardiovascular Development and Disease, vol. 5, no. 4, p. 50, 2018.

[3] M. R. Rosen, R. B. Robinson, P. R. Brink, and I. S. Cohen, "The road to biological pacing," Nature Reviews Cardiology, vol. 8, no. 11 , pp. 656-666, 2011.

[4] W. M. Hoogaars, A. Engel, J. F. Brons et al., "Tbx3 controls the sinoatrial node gene program and imposes pacemaker function on the atria," Genes \& Development, vol. 21, no. 9, pp. 1098-1112, 2007.

[5] R. J. Blaschke, N. D. Hahurij, S. Kuijper et al., "Targeted mutation reveals essential functions of the homeodomain transcription factor Shox2 in sinoatrial and pacemaking development," Circulation, vol. 115, no. 14, pp. 1830-1838, 2007.

[6] C. Wiese, T. Grieskamp, R. Airik et al., "Formation of the sinus node head and differentiation of sinus node myocardium are independently regulated by Tbx18 and Tbx3," Circulation Research, vol. 104, no. 3, pp. 388-397, 2009.

[7] D. DiFrancesco, "The role of the funny current in pacemaker activity," Circulation Research, vol. 106, no. 3, pp. 434-446, 2010 .
[8] M. L. Bakker, G. J. J. Boink, B. J. Boukens et al., “T-box transcription factor TBX3 reprogrammes mature cardiac myocytes into pacemaker-like cells," Cardiovascular Research, vol. 94, no. 3, pp. 439-449, 2012.

[9] H. F. Tse, T. Xue, C. P. Lau et al., "Bioartificial sinus node constructed via in vivo gene transfer of an engineered pacemaker HCN channel reduces the dependence on electronic pacemaker in a sick-sinus syndrome model," Circulation, vol. 114, no. 10, pp. 1000-1011, 2006.

[10] M. Choudhury, N. Black, A. Alghamdi et al., "TBX18 overexpression enhances pacemaker function in a rat subsidiary atrial pacemaker model of sick sinus syndrome," The Journal of Physiology, vol. 596, no. 24, pp. 6141-6155, 2018.

[11] N. Kapoor, W. Liang, E. Marbán, and H. C. Cho, "Direct conversion of quiescent cardiomyocytes to pacemaker cells by expression of Tbx18," Nature Biotechnology, vol. 31, no. 1, pp. 54-62, 2013.

[12] K. Oshita, M. Itoh, S. Hirashima et al., "Ectopic automaticity induced in ventricular myocytes by transgenic overexpression of HCN2," Journal of Molecular and Cellular Cardiology, vol. 80, pp. 81-89, 2015.

[13] M. R. Rosen, "Gene therapy and biological pacing," The New England Journal of Medicine, vol. 371, no. 12, pp. 1158-1159, 2014.

[14] Y. F. Hu, J. F. Dawkins, H. C. Cho, E. Marban, and E. Cingolani, "Biological pacemaker created by minimally invasive somatic reprogramming in pigs with complete heart block," Science Translational Medicine, vol. 6, no. 245, article 245ra94, 2014.

[15] Y. Li, M. Yang, G. Zhang et al., “Transcription factor TBX18 promotes adult rat bone mesenchymal stem cell differentiation to biological pacemaker cells," International Journal of Molecular Medicine, vol. 41, no. 2, pp. 845-851, 2018.

[16] H. Xiao, Y. J. Yang, Y. Z. Lin, S. Peng, S. Lin, and Z. Y. Song, "Transcription factor Tbx18 induces the differentiation of c$\mathrm{kit}^{+}$canine mesenchymal stem cells (cMSCs) into SAN-like pacemaker cells in a co-culture model in vitro," American Journal of Translational Research, vol. 10, no. 8, pp. 25112528, 2018

[17] A. M. Gorabi, S. Hajighasemi, H. A. Tafti et al., "TBX18 transcription factor overexpression in human-induced pluripotent stem cells increases their differentiation into pacemakerlike cells," Journal of Cellular Physiology, vol. 234, no. 2, pp. 1534-1546, 2019.

[18] V. Ionta, W. Liang, E. H. Kim et al., "SHOX2 overexpression favors differentiation of embryonic stem cells into cardiac pacemaker cells, improving biological pacing ability," Stem Cell Reports, vol. 4, no. 1, pp. 129-142, 2015.

[19] J. J. Jung, B. Husse, C. Rimmbach et al., "Programming and isolation of highly pure physiologically and pharmacologically functional sinus-nodal bodies from pluripotent stem cells," Stem Cell Reports, vol. 2, no. 5, pp. 592-605, 2014.

[20] A. Barbuti and R. B. Robinson, "Stem cell-derived nodal-like cardiomyocytes as a novel pharmacologic tool: insights from sinoatrial node development and function," Pharmacological Reviews, vol. 67, no. 2, pp. 368-388, 2015.

[21] D. Mushahary, A. Spittler, C. Kasper, V. Weber, and V. Charwat, "Isolation, cultivation, and characterization of human mesenchymal stem cells," Cytometry Part A, vol. 93, no. 1, pp. 19-31, 2018. 
[22] E. E. Verheijck, A. Wessels, A. C. G. van Ginneken et al., "Distribution of atrial and nodal cells within the rabbit sinoatrial node: models of sinoatrial transition," Circulation, vol. 97, no. 16, pp. 1623-1631, 1998.

[23] F. Greulich, M. O. Trowe, A. Leffler, C. Stoetzer, H. F. Farin, and A. Kispert, "Misexpression of Tbx18 in cardiac chambers of fetal mice interferes with chamber-specific developmental programs but does not induce a pacemaker-like gene signature," Journal of Molecular and Cellular Cardiology, vol. 97, pp. 140-149, 2016.

[24] E. G. Lakatta and V. A. Maltsev, "Reprogramming paces the heart," Nature Biotechnology, vol. 31, no. 1, pp. 31-32, 2013.

[25] E. G. Lakatta, V. A. Maltsev, and T. M. Vinogradova, "A coupled SYSTEM of intracellular $\mathrm{Ca}^{2+}$ clocks and surface membrane voltage clocks controls the timekeeping mechanism of the heart's pacemaker," Circulation Research, vol. 106, no. 4, pp. 659-673, 2010.

[26] P. A. Schweizer, F. F. Darche, N. D. Ullrich et al., "Subtypespecific differentiation of cardiac pacemaker cell clusters from human induced pluripotent stem cells," Stem Cell Research \& Therapy, vol. 8, no. 1, p. 229, 2017.

[27] L. Chen, Z. J. Deng, J. S. Zhou et al., “Tbx18-dependent differentiation of brown adipose tissue-derived stem cells toward cardiac pacemaker cells," Molecular and Cellular Biochemistry, vol. 433, no. 1-2, pp. 61-77, 2017.

[28] T. Xue, C. W. Siu, D. K. Lieu, C. P. Lau, H. F. Tse, and R. A. Li, "Mechanistic role of $I_{\mathrm{f}}$ revealed by induction of ventricular automaticity by somatic gene transfer of gating-engineered pacemaker (HCN) channels," Circulation, vol. 115 , no. 14, pp. 1839-1850, 2007.

[29] S. Herrmann, J. Stieber, G. Stöckl, F. Hofmann, and A. Ludwig, "HCN4 provides a 'depolarization reserve' and is not required for heart rate acceleration in mice," The EMBO Journal, vol. 26, no. 21, pp. 4423-4432, 2007.

[30] A. Milano, A. M. Vermeer, E. M. Lodder et al., "HCN4 mutations in multiple families with bradycardia and left ventricular noncompaction cardiomyopathy," Journal of the American College of Cardiology, vol. 64, no. 8, pp. 745-756, 2014.

[31] P. A. Schweizer, J. Schröter, S. Greiner et al., "The symptom complex of familial sinus node dysfunction and myocardial noncompaction is associated with mutations in the HCN4 channel," Journal of the American College of Cardiology, vol. 64, no. 8, pp. 757-767, 2014.

[32] C. Jun, Z. Zhihui, W. Lu et al., "Canine bone marrow mesenchymal stromal cells with lentiviral mHCN4 gene transfer create cardiac pacemakers," Cytotherapy, vol. 14, no. 5, pp. 529$539,2012$.

[33] W. Lu, N. Yaoming, R. Boli et al., "mHCN4 genetically modified canine mesenchymal stem cells provide biological pacemaking function in complete dogs with atrioventricular block," Pacing and Clinical Electrophysiology, vol. 36, no. 9, pp. 1138-1149, 2013.

[34] M. Baruscotti, A. Bucchi, C. Viscomi et al., "Deep bradycardia and heart block caused by inducible cardiac-specific knockout of the pacemaker channel gene Hcn4," Proceedings of the National Academy of Sciences of the United States of America, vol. 108, no. 4, pp. 1705-1710, 2011.

[35] R. X. Liu, Y. L. Wang, H. B. Li, N. N. Wang, M. J. Bao, and L. Y. $\mathrm{Xu}$, "Comparative study between original and traditional method in establishing a chronic sinus node damage model in rabbit," Journal of Applied Physiology, vol. 113, no. 11, pp. 1802-1808, 2012.
[36] H. B. Zhong, T. J. Wang, G. L. Lian, C. S. Xu, H. J. Wang, and L. D. Xie, "Development of a rat model of sick sinus syndrome using pinpoint press permeation," BioMed Research International, vol. 2018, Article ID 7487324, 7 pages, 2018.

[37] W. Zhang, B. Zhu, J. Ren et al., "Two methods for modeling of sick sinus syndrome in rats: ischemia reperfusion and sodium hydroxide induced injury," Biomedicine \& Pharmacotherapy, vol. 111, pp. 778-784, 2019. 


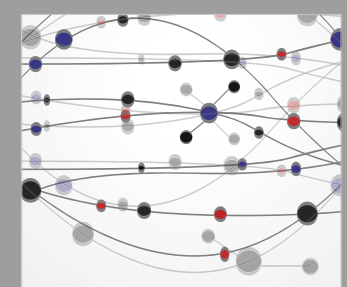

The Scientific World Journal
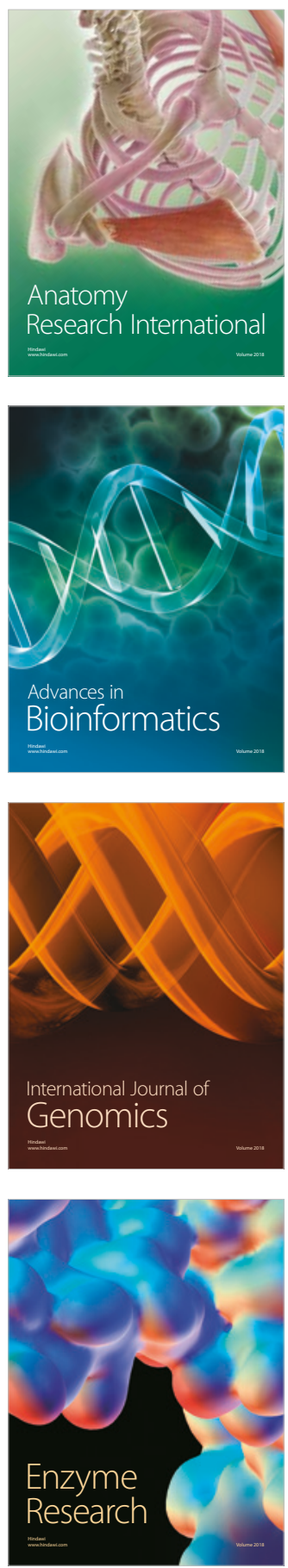
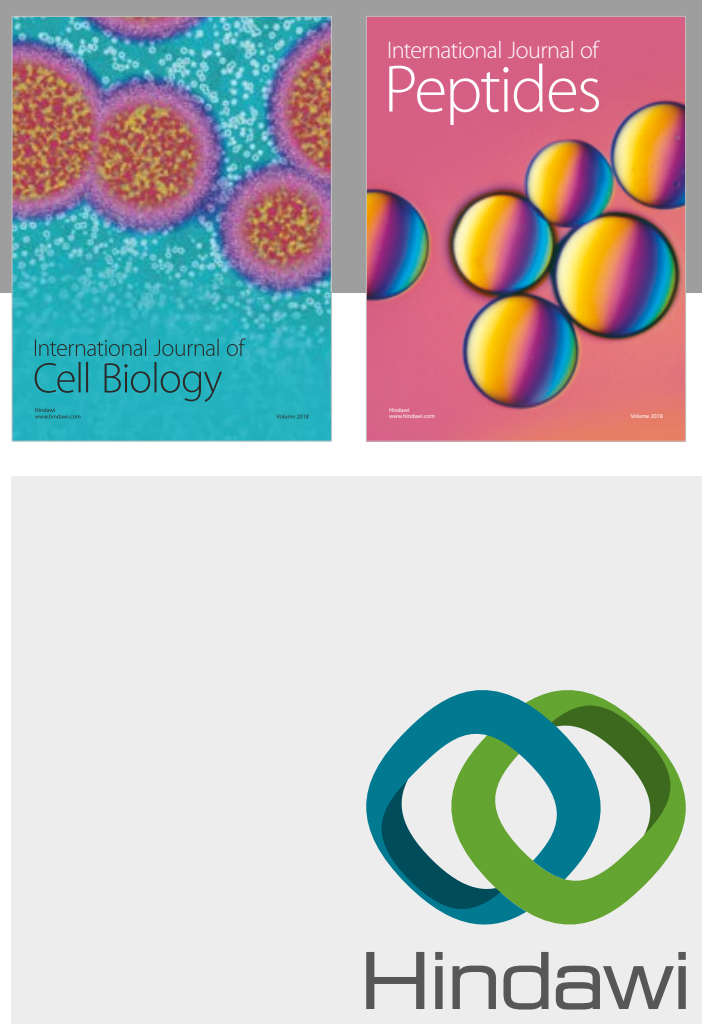

Submit your manuscripts at

www.hindawi.com
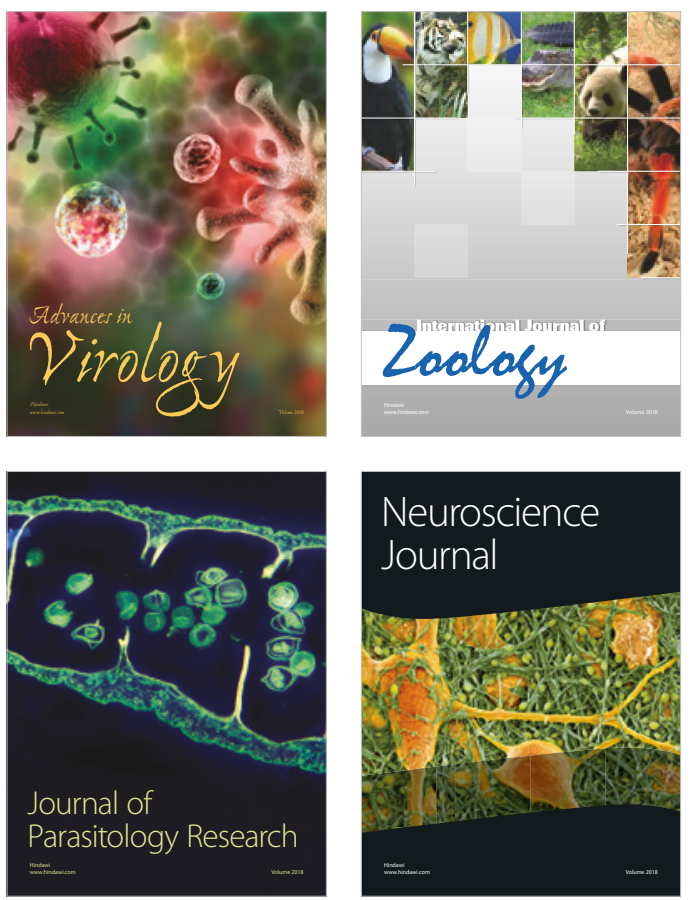
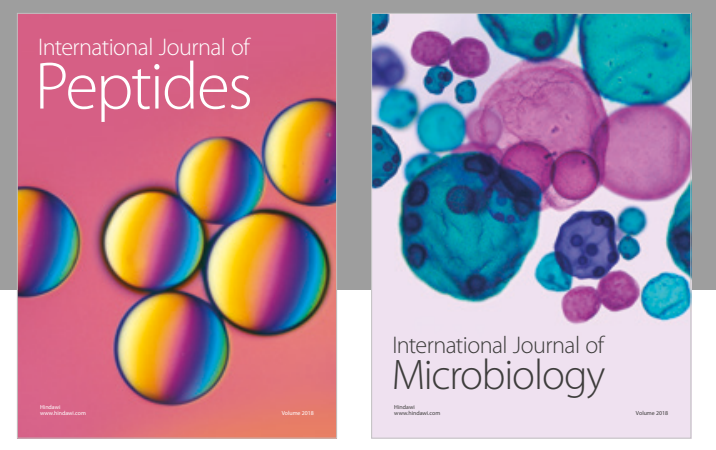

nternational Journal of Microbiology
Journal of
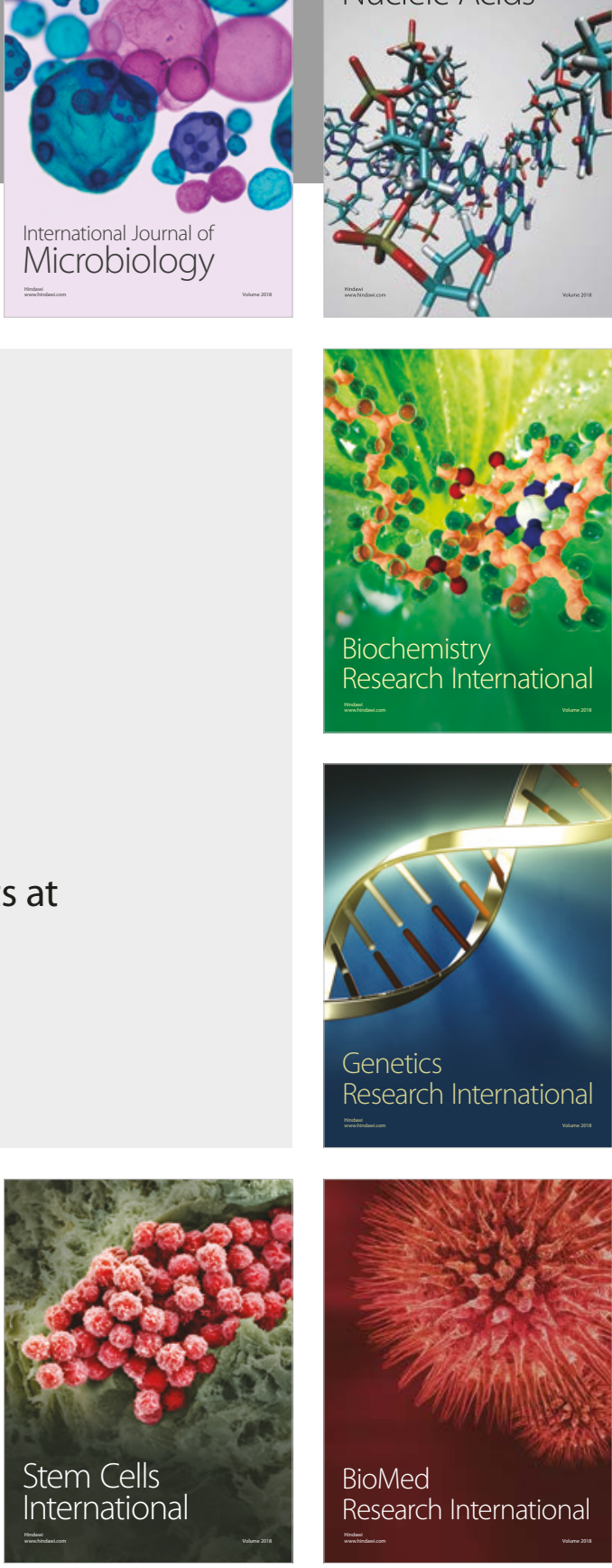
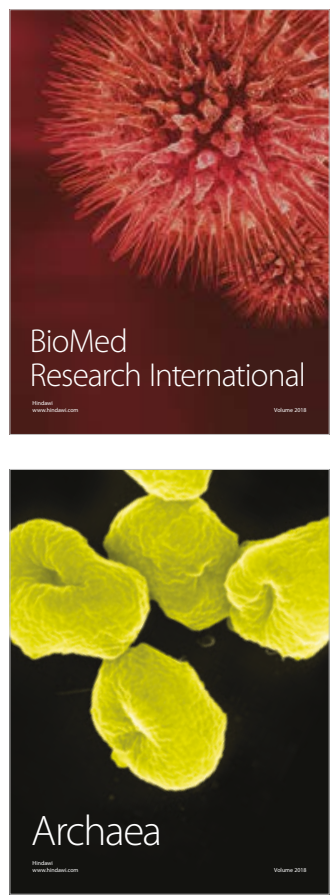